Research Article

\title{
Optimal Allocation of Shared Manufacturing Resources Based on Bilevel Programming
}

\author{
Peng Liu $\mathbb{D}$, Caiyun Liu, and Xiaoling Wei \\ School of Management, Shenyang University of Technology, Shenyang 110870, China \\ Correspondence should be addressed to Peng Liu; liup7802@163.com
}

Received 28 September 2021; Revised 26 November 2021; Accepted 30 November 2021; Published 23 December 2021

Academic Editor: Giulio E. Cantarella

Copyright (c) 2021 Peng Liu et al. This is an open access article distributed under the Creative Commons Attribution License, which permits unrestricted use, distribution, and reproduction in any medium, provided the original work is properly cited.

\begin{abstract}
In the shared manufacturing environment, on the basis of in-depth analysis of the shared manufacturing process and the allocation process of manufacturing resources, a bilevel programming model for the optimal allocation of manufacturing resources considering the benefits of the shared manufacturing platform and the rights of consumers is established. In the bilevel programming model, the flexible indicators representing the interests of the platform are the upper-level optimization target of the model and the Quality of Service (QoS) indicators representing the interests of consumers are the lower-level optimization goal. The weights of the upper indicators are determined by Analytic Hierarchy Process (AHP) and Improved Order Relation Analysis (Improved G1) combination weighting method and the bilevel programming model is solved by the Improved Fast Elitist NonDominated Sorting Genetic Algorithm (Improved NSGA-II). Finally, the effectiveness of the model is validated by a numerical example.
\end{abstract}

\section{Introduction}

As the foundation of the real economy and the pillar of the national economy, the manufacturing industry plays a vital role in promoting the vigorous development of the economy and improving people's living standards substantially. The rapid development and widespread application of technologies such as the digital economy and the industrial Internet have brought development opportunities for the manufacturing industry. As a new manufacturing mode, shared manufacturing has been formed. Based on the Internet platform, shared manufacturing focuses on the entire life cycle of manufacturing, gathers and integrates idle manufacturing resources of suppliers, and matches consumers' tasks intelligently and dynamically, to break the information asymmetry, to achieve benign cooperation between suppliers and demanders, and ultimately to achieve the reasonable allocation of shared manufacturing resources. Shared manufacturing has unparalleled advantages in the use of idle production capacity, the aggregation of scattered resources, and the allocation of tasks and resources.
Recently, shared manufacturing has received considerable attention in the literature. Ellen [1] proposes the concept of "shared manufacturing" in 1990. Ari Samadhi and Hoang [2] propose the sharing content in the complex manufacturing environment. Sheikhzadeh et al. [3] study two types of machine sharing configuration in manufacturing systems. Yu et al. [4] pay attention to the concept and definition of shared manufacturing. Jiang and Li [5] identify the feasible key-enabled technologies for configuring and running a shared factory and analyze the opportunities and challenges of launching the shared factory. Li et al. [6] compare the profit of the shared manufacturing equipment manufacturer under the service model with the gain of the purchase model and derive the conditions for choosing each business model. Xu et al. [7] investigate an online scheduling problem, where one manufacturer owning two parallel identical machines may lease a number of external machines to satisfy its jobs via a manufacturing resource sharing platform. Yu et al. [8] propose a shared memory framework based on blockchain to support the application of cyber-physical systems to expand the scope of resource sharing on a large scale both 
vertically and horizontally. Rožman et al. [9] propose an extensible framework for shared manufacturing based on blockchain and integrate blockchain technology into the concept of shared manufacturing by adopting a cross-chain solution. He et al. [10] present the sharing model of China's shared manufacturing platform and basic organizational architecture of manufacturing capacity sharing and give the detailed sharing process of distributed maintenance management platform. Li and Jiang [11] propose a new enhanced self-organizing agent in the context of sustainable shared manufacturing. Wang et al. [12] construct the digital twindriven service model and design to coordinate cross-organizations resources to promote resource allocation in shared manufacturing. Ayala et al. [13] study the dynamic application of knowledge sharing in manufacturing companies, which depended on the type of collaboration and service object.

Next, we will present a brief review on the bilevel programming as follows. Bilevel programming has a wide range of applications in multiobjective problems, especially in solving resource allocation problems. Zhao and Wang [14] propose a bilevel programming decision-making model of resource allocation under cloud manufacturing. Chen et al. [15] propose an optimal resource configuration model based on bilevel programming, which has added both interests of demand and service parties and cloud platform operators. Zhao et al. [16] propose a bilevel programming model undertaking a full consideration of interest and psychology for both sides. Wang et al. [17] construct a bilevel programming model oriented to resource providers, service demanders, and cloud platform operators to solve the optimization of resource composition in cloud manufacturing environment. Su et al. [18] establish a bilevel programming model of manufacturing resource allocation on top of the assessment index system. Wu et al. [19] propose a service capability evaluation model of resource combination, which includes QoS and sustainability factors. Wang and Du [20] establish a nonlinear bilevel programming model with product design as the leader and its implementation as the follower. Luo and $\mathrm{Wu}$ [21] construct a bilevel programming model to maximize customer satisfaction and minimize enterprise operation cost. Wang et al. [22] construct a bilevel programming model for the hazmat transportation network optimization, in which the upper level aims to minimize the maximum total risk of the transportation network, and the lower level focuses on minimizing the robust cost of the hazmat carriers. Huang and Ji [23] propose a bilevel optimization model with the factors of delivery time and distance under potential delay risk. Dong et al. [24] considered the supply risk under different disaster scenarios, selected multiple suppliers to buffer supply risks, and built a bilevel programming model. Wang et al. [25] establish a resource optimization model with the least cost, the least time, and the most quality based on formal description of resource allocation problem in cloud manufacturing. Xu and $\mathrm{Li} \mathrm{[26]}$ built a multiobjective optimization allocation model of manufacturing resources for cloud manufacturing services. Gao et al. [27] propose an optimal allocation method of manufacturing resource based on improved genetic algorithm. Yang et al. [28] propose an extended Parametric Complementary Pivot (PCP) algorithm for linear bilevel programming.

Although the significant progress has been made in the above literature, some research gaps still need to be fulfilled. On the one hand, some scholars study resource allocation in cloud manufacturing environment, but few scholars consider the multiple parties involved in shared manufacturing environment. On the other hand, the established indicator systems are not comprehensive enough. In this paper, we use the idea of bilevel programming to analyze shared manufacturing resource allocation based on the indicator system that puts Quality of Service (QoS) indicators and flexible indicators in an equally important position. We consider a bilevel programming model for the optimal allocation of shared manufacturing resources considering the benefits of the shared manufacturing platform and the rights of consumers.

The rest of this paper is organized as follows: In Section 2 , it introduces the problem description. In Section 3, we construct the bilevel programming model. We propose the solution of the model in Section 4. Numerical example is given in Section 5. Finally, Section 6 provides conclusions.

\section{Resource Optimal Allocation Problem in Shared Manufacturing}

Shared manufacturing includes shared manufacturing platforms and participants. In the shared manufacturing environment, companies and individuals have multiple roles, both as providers and consumers. The sharing process of manufacturing resources involves the participation of three parties, namely, shared manufacturing providers, consumers, and platforms. It is completed by four chains, namely, the configuration chain, the service chain, the payment chain, and the monitoring chain, as shown in Figure 1. MT is manufacturing task, MR is manufacturing resource, SMT is the notation of submanufacturing task, and SMR is submanufacturing resource.

The configuration chain is dominated by a shared manufacturing platform. One party, as provider, provides resources with different functions or services to meet consumer's needs on the shared manufacturing platform, which is decomposed and encapsulated by the platform. One party, as consumer, submits tasks on the shared manufacturing platform, which is rotted and recorded by the platform according to the granularity matching the task. Resources and functions are configured on this basis. After the configuration chain is completed, the service chain starts to operate. The service chain is dominated by providers and consumers. Consumers communicate with providers in the form of "Peer to peer," and providers also provide services to consumers in "Peer to peer." The service chain is completed, and the payment chain starts to operate. The payment chain is dominated by shared manufacturing platform, providers, and consumers. Once the needs of the task are met, the provider will submit payment request to the platform, and the platform will then issue payment notification to the consumer in the next step. After the consumer confirms the 


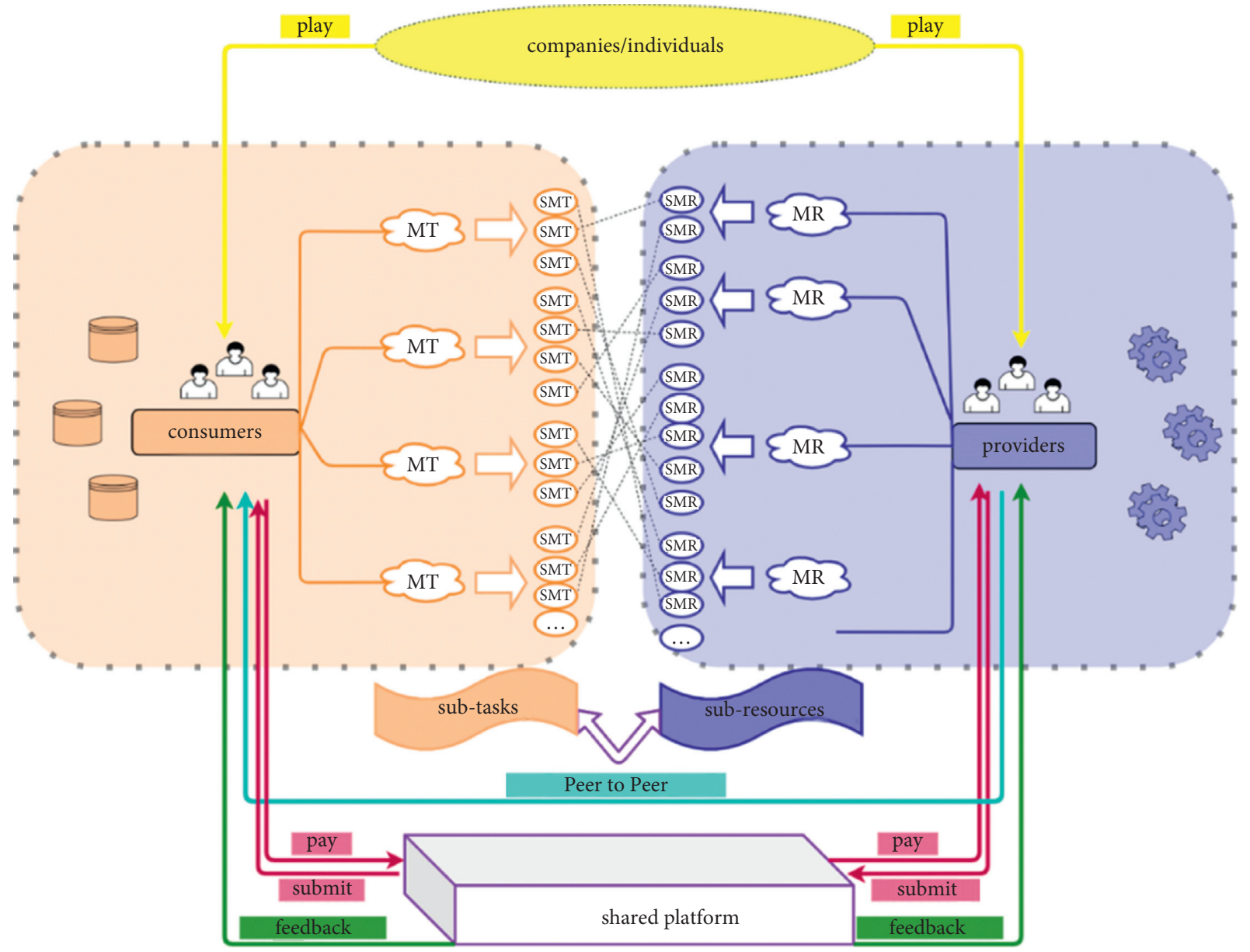

FIGURE 1: Shared process of shared manufacturing resources.

service, the payment is made to the platform, and the platform charges and finally delivers it to the provider. The monitoring chain is always involved in the whole operation of the configuration chain, the service chain, and the payment chain. The monitoring chain is dominated by shared manufacturing platform. The platform monitors the whole process of both providers and consumers and provides timely feedback to the information demanders to ensure that the task needs of the consumers can be met, while safeguarding the interests of the providers.

The shared manufacturing resource allocation in the configuration chain is the core of the whole sharing process. Shared Manufacturing Resource Allocation is a process in which a shared platform reasonably allocates the resource service of the provider for various manufacturing tasks submitted by consumers under multiobjective functions and multiconstraint conditions. The platform decomposes the manufacturing tasks submitted by consumers into four levels of tasks: product level, part level, process segment level, and process level. These four levels of tasks will be encapsulated as subtasks. The platform decomposes the supplier's resources into four granularities that match the tasks, namely, enterprise level, workshop level, unit level, and equipment level. These four levels of resources will be encapsulated as subresources. Finally, shared platform matches the candidate manufacturing resource set for the subtasks according to the consumer requirements, as can be seen from Figure 2.

In the process of shared manufacturing resource allocation, the shared platform plays a leading role, and the healthy development of the platform is the foundation and the focus, so it is worth paying attention to the flexible indicators. Additionally, the consumers, as the initiator of the task, focus on whether the commission can meet expectations, so the Quality of Service (QoS) indicators are also worthy of attention. Constructing a reasonable evaluation indicator system and establishing a resource optimal allocation model are crucial to the study of the shared manufacturing resource allocation.

\section{Model Establishment}

The shared manufacturing resource allocation is a complex multiobjective optimization problem, and the optimal allocation of shared manufacturing resources involves the multiple restricted interests of resource providers, consumers, and shared manufacturing platforms. Therefore, a bilevel programming model is employed to solve the problem. The general model of bilevel programming can be formulated as 


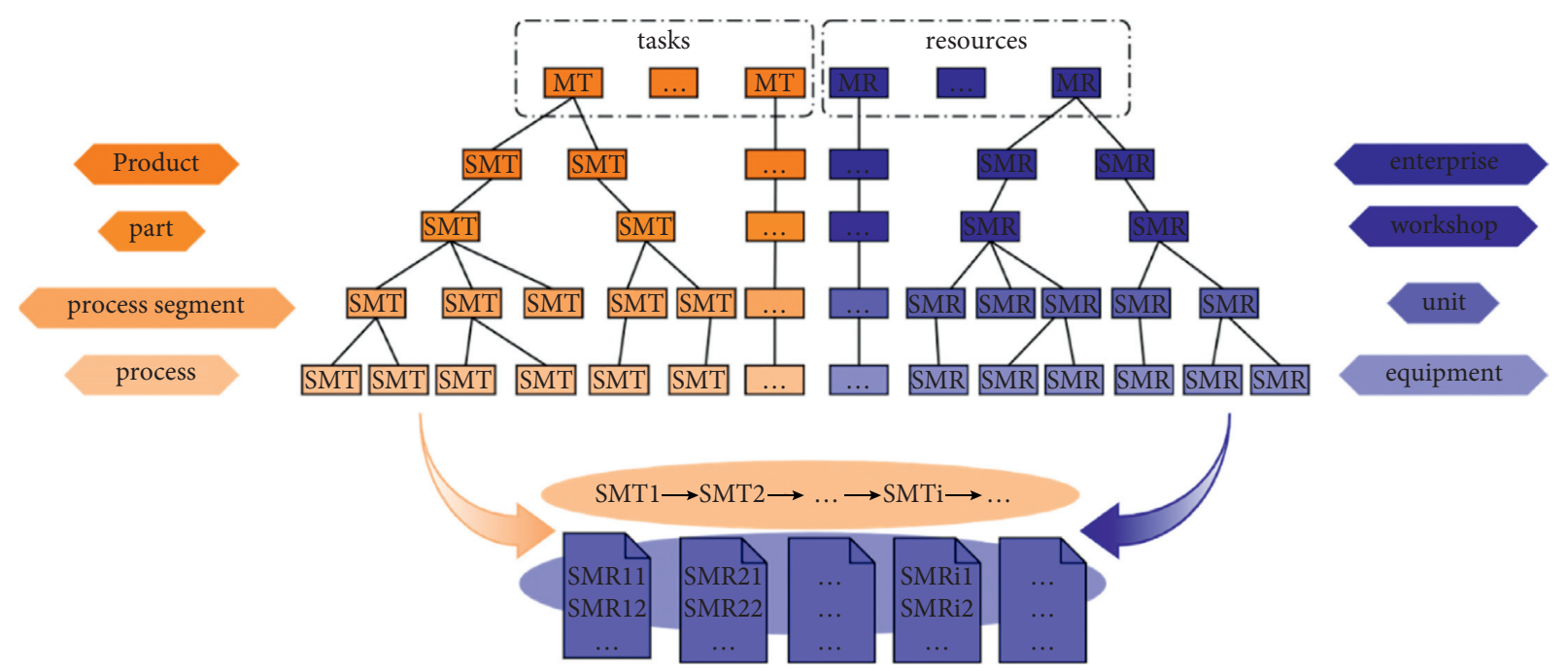

Figure 2: The allocation of shared manufacturing resources and tasks.

$$
\begin{gathered}
(U) \min F(x, y), \\
\text { s.t. } G(x, y) \leq 0, \\
(L) \min f(x, y), \\
\text { s.t. } g(x, y) \leq 0 .
\end{gathered}
$$

In the bilevel programming model, there are two levels in total, where $(U)$ is the upper-level programming, and $(L)$ is the lower-level programming. In the upper-level programming, $F$ represents the objective function, $x$ is the decision variable, and $G$ is the constraint condition of $x$. Similarly, in the lower-level programming, $f$ is the objective function, $y$ is the decision variable, and $g$ is the constraint condition of $y$. There is a functional relationship, that is, $y=y(x)$, between decision variables in upper-level programming and lowerlevel programming.

The upper- and lower-level optimization of the bilevel programming model are relatively independent, but they also affect and restrict each other. In the shared manufacturing environment, the optimal allocation of resources should consider the interests of shared platform and shared consumers. Therefore, the bilevel programming model for the optimal allocation of shared manufacturing resources is established with reliability, credit, risk management, and service evaluation as upper evaluation indicators and cost, time, and quality qualification rate as lower evaluation indicators, as shown in Figure 3. The notations involved in the model are defined in Table 1.

3.1. Upper-Level Programming. The shared manufacturing platform is regarded as the upper decision-maker of the bilevel model in optimal allocation of shared manufacturing resources. The development of the platform is based on the trust of all parties. Reliability indicator can help the platform choose superior resources in terms of materials and technology, thus laying the foundation for the completion of the task. The credibility indicator facilitates the platform to screen out resources with a higher level of historical evaluation to process tasks. The risk management indicators are the platform's ability to evaluate resources in response to changes. The service evaluation indicator is the consumer's evaluation of the whole service process. All four indicators can directly reflect the consumer's trust in the shared manufacturing platform, so reliability, credibility, risk management, and service evaluation represent the benefits of the shared manufacturing platform.

3.1.1. Reliability. The reliability indicator (RE) includes three aspects: technological advancement, maintainability, and resource quality assurance. Among them, technological advancement refers to the evaluation of the manufacturing technology level used in the processing of resources; maintainability refers to the evaluation of maintenance measures for semifinished and finished products during task processing; resource quality assurance refers to the evaluation of the quality level of resources used to complete the task and the evaluation of the integrity of the system that guarantees the quality level. Formula (2) is employed to calculate the reliability indicator.

$$
\mathrm{RE}=\frac{\sum_{i=1}^{n} \sum_{j=1}^{m_{i}} u_{i j} r e(i j)}{n}
$$

3.1.2. Credibility. The credibility indicator (CR) includes processing efficiency, quality level, and resource utilization. Processing efficiency refers to the time required to complete the required number of tasks; quality level refers to the quality level of resources; resource utilization refers to the efficiency of converting resources into products. Formula (3) is employed to calculate the credibility indicator.

$$
\mathrm{CR}=\frac{\sum_{i=1}^{n} \sum_{j=1}^{m_{i}} u_{i j} c r(i j)}{n} \text {. }
$$




\section{Bi-level programming model $\quad$ Decision-making body}

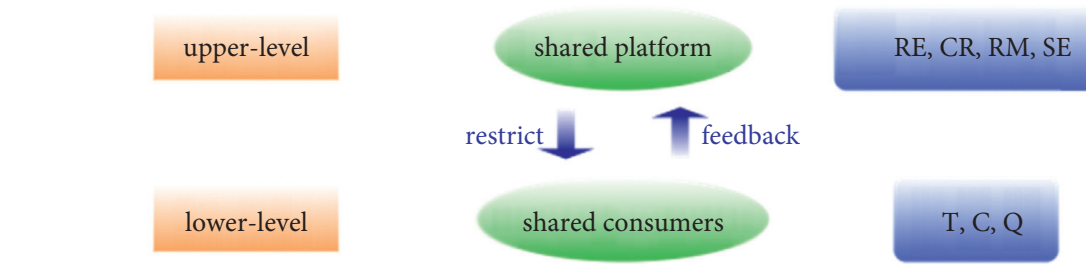

RE, CR, RM, SE

FIGURE 3: Bilevel programming model for optimal allocation of shared manufacturing resources.

TABLE 1: Notations.

\begin{tabular}{|c|c|}
\hline Notations & Description \\
\hline$f$ & The upper-level objective of shared manufacturing resource allocation \\
\hline$F$ & The lower-level objective of shared manufacturing resource allocation \\
\hline $\mathrm{RE}$ & The total reliability evaluation for completing all tasks \\
\hline $\mathrm{CR}$ & The total credibility evaluation for completing all tasks \\
\hline $\mathrm{RM}$ & The total risk management evaluation for completing all tasks \\
\hline SE & The total service evaluation for completing all tasks \\
\hline C & The total manufacturing cost for completing all tasks \\
\hline$T$ & The total manufacturing time for completing all tasks \\
\hline Q & The total quality qualification rate for completing all tasks \\
\hline$n$ & The number of tasks \\
\hline$m_{i}$ & The number of candidate resource for the $i$ th task \\
\hline$O_{i j}$ & The $j$ th candidate shared manufacturing resource for $i$ th task \\
\hline$O_{(i+1) k}$ & The $k$ th candidate shared manufacturing resource for $(i+1)$ th task \\
\hline$r e(i j)$ & The manufacturing reliability evaluation for $O_{i j}$ completing the $i$ th task \\
\hline $\operatorname{cr}(i j)$ & The manufacturing credibility evaluation for $O_{i j}$ completing the $i$ th task \\
\hline$r m(i j)$ & The manufacturing risk management evaluation for $O_{i j}$ completing the $i$ th task \\
\hline$s e(i j)$ & The manufacturing service evaluation for $O_{i j}$ completing the $i$ th task \\
\hline$C_{1}(i j)$ & The processing cost for $O_{i j}$ completing the $i$ th task \\
\hline$C_{2}(i j)$ & The labor cost for $O_{i j}$ completing the $i$ th task \\
\hline$C_{3}(i j,(i+1) k)$ & The linking cost for $O_{i j}$ and $O_{(i+1) k}$ \\
\hline$T_{1}(i j)$ & The processing time for $O_{i j}$ completing the $i$ th task \\
\hline$T_{2}(i j)$ & The wastage time for $O_{i j}$ completing the $i$ th task \\
\hline$T_{3}(i j,(i+1) k)$ & The linking time for $O_{i j}$ and $O_{(i+1) k}$ \\
\hline$q(i j)$ & The quality qualification rate for $O_{i j}$ completing the $i$ th task \\
\hline$C_{\max }$ & The maximum manufacturing cost for completing all tasks \\
\hline$T_{\max }$ & The maximum manufacturing time for completing all tasks \\
\hline$Q_{\min }$ & The minimum manufacturing quality qualification rate for completing all tasks \\
\hline$\omega_{1}$ & The weight coefficient for reliability evaluation of completing all tasks \\
\hline$\omega_{2}$ & The weight coefficient for credibility evaluation of completing all tasks \\
\hline$\omega_{3}$ & The weight coefficient for risk management evaluation of completing all tasks \\
\hline$\omega_{4}$ & The weight coefficient for service evaluation of completing all tasks \\
\hline$u_{i j}$ & The selection coefficient for $O_{i j}$ \\
\hline$u_{(i+1) k}$ & The selection coefficient for $O_{(i+1) k}$ \\
\hline
\end{tabular}

3.1.3. Risk Management. The risk management indicator (RM) includes the functional diversity, recoverability, and quantity of alternative resources. Functional diversity refers to the extensive use of resources; recoverability refers to the ability to repair failed resources to the specified requirements, that is, the time, cost, and labor required for the process; quantity of alternative resources refers to the amount of resource substitutes with the same manufacturing function. Formula (4) is employed to calculate the risk management indicator.

$$
\mathrm{RM}=\frac{\sum_{i=1}^{n} \sum_{j=1}^{m_{i}} u_{i j} r m(i j)}{n} .
$$

3.1.4. Service Evaluation. The service evaluation indicator (SE) includes service attitude, service capability, and service level. Service attitude refers to the communication between consumers and providers about task requirements before processing, the processing attitude of providers during 
processing, and the negotiation of delivery after processing; service capability refers to the level of business and professionalism shown in the entire service process; service level refers to the degree to which the delivered tasks meet the requirements. Formula (5) is employed to calculate the service evaluation indicator.

$$
\mathrm{SE}=\frac{\sum_{i=1}^{n} \sum_{j=1}^{m_{i}} u_{i j} s e(i j)}{n} .
$$

3.1.5. Upper-Level Optimization Objective. In the bilevel programming model, the objective function of the upperlevel is formula (6). It includes four parts, namely, reliability, credibility, risk management, and service evaluation. The relationship among all weights could be expressed as formula (7).

$$
\begin{gathered}
\max f=\omega_{1} \mathrm{RE}+\omega_{2} \mathrm{CR}+\omega_{3} \mathrm{RM}+\omega_{4} \mathrm{SE}, \\
\omega_{1}+\omega_{2}+\omega_{3}+\omega_{4}=1 .
\end{gathered}
$$

3.2. Lower-Level Programming. In optimal allocation of shared manufacturing resources, the shared consumer is regarded as the lower-level decision-maker of the bilevel model. The cost is the first concern of consumers. The time is related to whether the provider can complete the consumer's task at the appointed time or even ahead of time. The quality qualification rate of resources largely determines the quality of the entire task. Therefore, cost, time, and quality qualification rate are on behalf of the interests of consumers.

3.2.1. Cost. The cost indicator $(C)$ is composed of the processing cost $\left(C_{1}\right)$, the labor cost $\left(C_{2}\right)$, and the linking cost $\left(C_{3}\right)$. The processing cost refers to the cost of raw materials and mechanical equipment consumed during processing; the labor cost includes wage subsidies for workshop workers and managers; the linking cost between tasks refers to the cost of transferring transportation to the next processing task after the current processing task is completed. Formula (8) is employed to calculate total cost.

$$
\begin{aligned}
C= & \sum_{i=1}^{n}\left[\sum_{j=1}^{m_{i}} u_{i j}\left(C_{1}(i j)+C_{2}(i j)\right)\right] \\
& +\sum_{i=1}^{n-1}\left[\sum_{j=1}^{m_{i}} \sum_{k=1}^{m_{i+1}} u_{i j} u_{(i+1) k} C_{3}(i j,(i+1) k)\right] .
\end{aligned}
$$

3.2.2. Time. The time indicator $(T)$ includes the task processing time $\left(T_{1}\right)$, the wastage time $\left(T_{2}\right)$, and the circulation time $\left(T_{3}\right)$. The processing time refers to the actual processing time of the task; the wastage time refers to the time to complete the invalid task; the circulation time between tasks refers to the time, which takes resources to transfer to the next task after completing the corresponding task. Formula (9) is employed to calculate total time.

$$
\begin{aligned}
T= & \sum_{i=1}^{n}\left[\sum_{j=1}^{m_{i}} u_{i j}\left(T_{1}(i j)+T_{2}(i j)\right)\right] \\
& +\sum_{i=1}^{n-1}\left[\sum_{j=1}^{m_{i}} \sum_{k=1}^{m_{i+1}} u_{i j} u_{(i+1) k} T_{3}(i j,(i+1) k)\right] .
\end{aligned}
$$

3.2.3. Quality Qualification Rate. The quality qualification rate indicator $(Q)$ refers to the situation that the quality of the finished product meets the requirements during the processing. Formula (10) is employed to calculate the quality qualification rate indicator.

$$
\mathrm{Q}=\frac{\sum_{i=1}^{n} \sum_{j=1}^{m_{i}} u_{i j} q(i j)}{n} .
$$

3.2.4. Lower-Level Optimization Objectives. Formula (11) represents the lower objective function in the bilevel programming model.

$$
\max F=\left(\frac{C_{\max }}{C}, \frac{T_{\max }}{T}, \frac{Q}{Q_{\min }}\right)^{T} .
$$

Among them, $C_{\max }$ represents the maximum payment cost, $T_{\max }$ represents the longest delivery time, and similarly, $Q_{\min }$ represents the minimum quality qualification rate.

3.3. Bilevel Programming Model. The bilevel programming model for the optimal allocation of shared manufacturing resources is shown as

$$
\begin{aligned}
(U) \max f & =\omega_{1} \mathrm{RE}+\omega_{2} \mathrm{CR}+\omega_{3} \mathrm{RM}+\omega_{4} \mathrm{SE} \\
& \sum_{j=1}^{m_{i}} u_{i j}=1, \quad i=1,2,3,4,5, \\
& \omega_{1}+\omega_{2}+\omega_{3}+\omega_{4}=1, \\
(L) \max F= & \left(\frac{C_{\max }}{C}, \frac{T_{\max }}{T}, \frac{Q}{Q_{\min }}\right)^{T}, \\
u_{i j}= & \left\{\begin{array}{ll}
1, & \text { if } O_{i j} \text { is selected, } \\
0, & \text { otherwise, }
\end{array} u_{(i+1) k}= \begin{cases}1, & \text { if } O_{(i+1) k} \text { is selected, } \\
0, & \text { otherwise. }\end{cases} \right.
\end{aligned}
$$

In the bilevel programming model, the lower-level programming optimizes its objective under the constraint of the upper-level programming and returns the optimal solution to the upper-level programming. The upper-level planning is further optimized according to its own goals based on the solution of the lower-level programming. Finally, the optimal solution of the problem is obtained. The interest of the shared platform is taken as the upper-level optimization goal and the interest of the shared consumers as the lower-level optimization goal. The interest of the shared platform is taken into account, while the interest of the shared consumers is guaranteed. 


\section{Model Algorithm}

The optimal allocation of shared manufacturing resources is a typical NP-hard problem, which is usually solved by intelligent algorithm. The optimal allocation of resources in the shared manufacturing environment is highly compatible with the idea of survival of the fittest in the principle of genetic algorithm. In contrast, the Fast Elitist Non-Nominated Sorting Genetic Algorithm (NSGA-II) has made improvements on the basis of the genetic algorithm, which makes the optimization faster, so it is reasonable to use NSGA-II to solve the bilevel programming model. In addition, Analytic Hierarchy Process (AHP) and Improved Order Relation Analysis (Improved G1) combination weighting method are used to determine the weight of upper-level indicators, which makes the weight assignment of the four indicators more scientific and reasonable.

\subsection{AHP and Improved G1 Combination Weighting Method.}

There are many indicators in the upper-level evaluation indicator system in the shared manufacturing environment. The general index weighting methods either rely on the subjective experience to simply weight the relationship among the indexes or use the historical data to weight objectively. It is difficult to effectively quantify the manufacturing resource evaluation indicators. AHP and Improved G1 combination weighting method combines objective empowerment method and subjective empowerment method, which makes it accurate, objective, and scientific.

4.1.1. AHP Subjective Weighting Method. In the comprehensive evaluation problem, Analytic Hierarchy Process is a commonly used subjective weighting method with a wide range of applications. Its basic steps could be briefly summarized as follows:

Step 1. Construct the judgment matrix of the evaluation index;

Step 2. Calculate Consistency Index and Consistency Ratio, and check whether the judgment matrix is consistent according to the result;

Step 3. Calculate the maximum eigenvalue and its corresponding eigenvector. The eigenvector is the weight vector of the corresponding evaluation index calculated by the AHP method.

4.1.2. Improved G1 Objective Weighting Method. In the Improved G1 method, the ratio of variation coefficient among indexes is used to replace the subjective evaluation proportion of experts, which makes it an objective weighting method.

The steps of the Improved G1 method are summarized as follows:

Step 1. Standardization of indicators:

$$
Y_{i j}=\frac{X_{i j}-\min \left(X_{i j}\right)}{\max \left(X_{i j}\right)-\min \left(X_{i j}\right)} .
$$

In order to unify the dimensions, the indicators are standardized. The standardized calculation is shown as formula (13).

Step 2. Coefficient of variation:

$$
V_{j}=\frac{\sum_{i=1}^{n} Y_{i j} / n}{\sqrt{(1 / n) \sum_{i=1}^{n}\left(Y_{i j}-\left(\sum_{i=1}^{n} Y_{i j} / n\right)\right)^{2}}} .
$$

Formula (14) is employed to calculate the coefficient of variation of the indicator.

Step 3. Indicator weight:

$$
\begin{aligned}
& r_{j}= \begin{cases}1, & V_{j-1}<V_{j}, \\
\frac{V_{j-1}}{V_{j}}, & V_{j-1} \geq V_{j},\end{cases} \\
& \omega_{j}^{[2]}=\left(1+\sum_{k=2}^{m} \prod_{j=k}^{m} r_{j}\right)^{-1}, \\
& \omega_{k-1}^{[2]}=r_{k} \omega_{k}^{[2]}, \quad k=j, j-1, \ldots, 2 .
\end{aligned}
$$

According to the coefficient of variation, formula (15) is employed to calculate the ratio $r_{j}$ of the relative importance of indicators. The weight of the $j$ th index is calculated as formula (16), and the weights of other indexes are calculated according to formula (17). Eventually, the Improved G1 objective weighting method determines the weights, that is, $\omega_{j}^{[2]}$.

\subsubsection{Combination Weighting}

$$
\omega_{j}=\frac{\omega_{j}^{[1]} \omega_{j}^{[2]}}{\sum_{j=1}^{n} \omega_{j}^{[1]} \omega_{j}^{[2]}} .
$$

The weights of the indexes are eventually calculated by AHP and Improved G1 combination weighting method, and the calculation formula is shown as formula (18).

4.2. Improved NSGA-II. In NSGA-II, parents are selected from the population according to the fitness value, and the genes of the selected parents are crossed and mutated to form offspring, and then a new population is selected according to the fitness of the offspring and the parents. With the repetition of this process, the fitness value of the individuals in the population will gradually increase and converge to the desired direction. In this paper, Improved NSGA-II of the multiobjective optimization algorithm is adopted. The individuals in the population are ranked using crowding operator and Pareto dominance and then gradually converge to the Pareto front in the iteration. Figure 4 


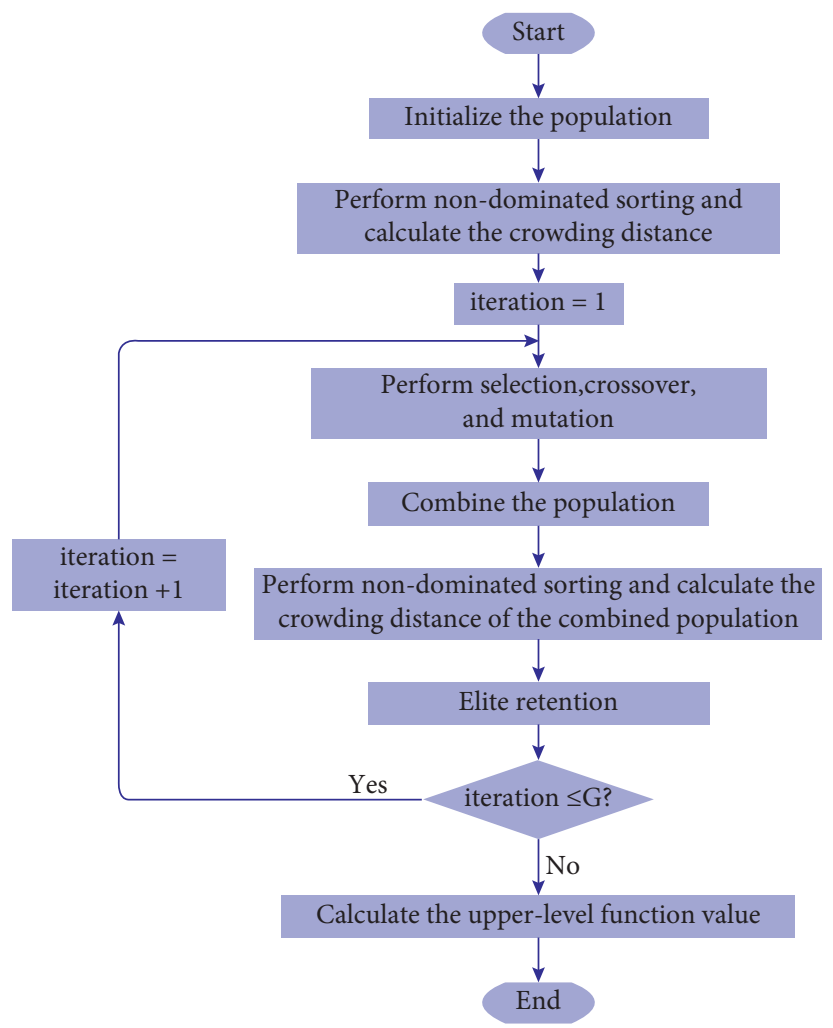

Figure 4: The process of Improved NSGA-II.

shows the flowchart of the Improved NSGA-II for solving the bilevel programming problem.

The solving steps are as follows:

Step 1. Initialize the population; set the parameters of the bilevel programming, namely, the number of generations, the population size, the crossover probability, and the mutation probability. The initial parent population P0 is randomly generated (the population size is NP).

Step 2. Sort the population; the combination of manufacturing resources in the shared manufacturing environment is coded to form the corresponding individual gene. For example, the resource allocation scheme $\mathrm{SMR}_{11}-\mathrm{SMR}_{22}-\mathrm{SMR}_{33}-\mathrm{SMR}_{42}-\mathrm{SMR}_{53}$ is coded as a gene $[1,2,3,2,3]$. The fitness values could be calculated by using formulas (8)-(10). Then, sort the population based on nondomination and crowding distance values.

Step 3. Perform selection, crossover, and mutation; the parent population $\mathrm{P} 0$ was selected, crossed, and mutated to generate the offspring population P01 (the population size is NP).
In selection, the reproduction operator is used to inherit the individuals with high fitness to the next generation population. The specific operation method is that, according to the nondominated ranking, individuals are selected from the first level downwards, and individuals at the same level choose the ones with a larger crowded distance.

In crossover, the arithmetic crossover operator acts on two individuals to produce two new individuals. The specific operation method is that the computer randomly generates a number $v$ between 0 and 1 . Then, formula (19) is employed to calculate the crossing coefficient. The offspring $\mathrm{Chromo}_{21}$ and $\mathrm{Chromo}_{22}$ were obtained by crossing $\mathrm{Chromo}_{11}$ and $\mathrm{Chromo}_{12}$ according to formula (20).

In mutation, the nonuniform mutation operator is used to perturb the original gene value randomly, and the population after the perturbation is used as the new gene value after mutation. The specific operation method is that a number $u$ between 0 and 1 is randomly generated by the computer, and the variation coefficient is calculated according to formula (21). $\mathrm{Chromo}_{1}$ was mutated by using formula (22) to obtain $\mathrm{chromo}_{2}$. 
After selection, crossover, and mutation, $\mathrm{P}_{01}$ is generated.

$$
\begin{aligned}
& \operatorname{gama}= \begin{cases}(2 v)^{\left(1 /\left(\mathrm{yita}_{1}+1\right)\right)-1}, & v<0.5 \\
1-[2(1-v)]^{\left(1 /\left(\mathrm{yita}_{1}+1\right)\right)}, & v \geq 0.5\end{cases} \\
& \left\{\begin{array}{l}
\text { chromo }_{21}=\left\lceil 0.5\left[(1+\text { gama }) \text { chromo }_{11}+(1-\text { gama }) \text { chromo }_{12}\right]\right\rceil, \\
\text { chromo }_{22}=\left\lceil 0.5\left[(1-\text { gama }) \text { chromo }_{11}+(1+\text { gama }) \text { chromo }_{12}\right]\right\rceil,
\end{array}\right. \\
& \text { delta }= \begin{cases}(2 u)^{(1 /(\text { yita2 }+1))-1}, & u<0.5, \\
1-[2(1-u)]^{(1 /(\text { yita } 2+1))}, & u \geq 0.5,\end{cases} \\
& \text { chromo }_{2}=\left\lceil\text { chromo }_{1}+\text { delta }\right\rceil .
\end{aligned}
$$

Step 4. Combine the population; the parent population $\mathrm{P} 0$ and the offspring population P01 are merged to form a combined population P02 (the population size is 2NP).

Step 5. Perform nondominated sorting and calculate the crowding distance of the combined population; fitness values of mixed population P02 were calculated according to formulas (8)-(10), and nondominated sorting was performed according to fitness value. In the meantime, it is to calculate the crowding distance of the combined population.

Step 6. Elite retention; according to the elite retention strategy, NP individuals are selected from the mixed population P02 to enter the next generation to form the population $\mathrm{P} 1$ (the population size is NP).

Step 7. Judge whether the loop is terminated; judge whether the iteration number is less than or equal to the maximum number of generations. If yes, then iteration $=$ iteration +1 , and skip to step 3; if not, then jump out of the loop. Repeat the above steps until the termination condition of the maximum genetic algebra is satisfied. The solution set of the lower objective function of the bilevel programming model for the optimal allocation of manufacturing resources is obtained.

Step 8. Calculate the upper-level function value.

The Pareto solution set obtained in step 6 is taken as the feasible solution set of the upper objective function of the bilevel programming model for the optimal allocation of manufacturing resources. Calculate the upper objective function value by using formulas (2)-(7).

\section{Numerical Example Analysis}

5.1. Calculation Example. Consumers publish the task requirements for cost, time, and quality qualification rate on the shared platform. Subsequently, the platform decomposes the task into five subtasks. The candidate manufacturing resources corresponding to these five subtasks are shown in
Table 2, the parameters such as the time and cost for the corresponding candidate resources are shown in Table 3 , and the linking cost and linking time between the subtasks are listed in Tables 4 and 5.

5.2. Example Solution. The judgment matrix of the four evaluation indicators in this calculation example is described as

$$
A=\left[\begin{array}{cccc}
1 & 3 & 5 & 7 \\
1 / 3 & 1 & 2 & 3 \\
1 / 5 & 1 / 2 & 1 & 2 \\
1 / 7 & 1 / 3 & 1 / 2 & 1
\end{array}\right] .
$$

The maximum eigenvalue of the judgment matrix is 4.0192. Consistency index is 0.0064 , and consistency ratio is 0.0071 , which are obviously less than 0.1 . The consistency of this matrix is acceptable. The corresponding weights of four indexes obtained by AHP method are $\omega_{1}^{[1]}=0.587166$, $\omega_{2}^{[1]}=0.217876, \omega_{3}^{[1]}=0.122786, \omega_{4}^{[1]}=0.072172$. According to formulas (13)-(17), the corresponding weights of the four indexes obtained by the Improved G1 method are $\omega_{1}^{[2]}=0.299442, \quad \omega_{2}^{[2]}=0.268492, \quad \omega_{3}^{[2]}=0.268492$, $\omega_{4}^{[2]}=0.163574$. Finally, according to formula (18), AHP and Improved G1 combination weighting method determines the reliability weight to be 0.63 , the credibility weight to be 0.21 , the risk management weight to be 0.12 , and the service evaluation weight to be 0.04 .

We use the software MATLAB R2018a to get the results. The parameters set in our calculation example are as follows. The number of generations is set as 200 , and the population size is 180 . In the meantime, the crossover probability and the mutation probability are set to 0.7 and 0.03 , respectively. Other parameters are set as $C_{\max }=1800, T_{\max }=200$, $Q_{\min }=0.60$.

A set of allocations of shared manufacturing resources is obtained by running. The duplicate data is deleted, and the final result is shown in Table 6. The plot of the final populations with the Maximum fitness value is shown in Figure 5. 
TABLE 2: Candidate resources for manufacturing subtasks.

\begin{tabular}{|c|c|c|c|c|c|}
\hline Manufacturing subtasks & $\mathrm{SMT}_{1}$ & $\mathrm{SMT}_{2}$ & $\mathrm{SMT}_{3}$ & $\mathrm{SMT}_{4}$ & $\mathrm{SMT}_{5}$ \\
\hline \multirow{4}{*}{ Candidate Manufacturing Resources } & $\mathrm{SMR}_{11}$ & $\mathrm{SMR}_{21}$ & $\mathrm{SMR}_{31}$ & $\mathrm{SMR}_{41}$ & $\mathrm{SMR}_{51}$ \\
\hline & $\mathrm{SMR}_{12}$ & $\mathrm{SMR}_{22}$ & $\mathrm{SMR}_{32}$ & $\mathrm{SMR}_{42}$ & $\mathrm{SMR}_{52}$ \\
\hline & $\mathrm{SMR}_{13}$ & $\mathrm{SMR}_{23}$ & $\mathrm{SMR}_{33}$ & $\mathrm{SMR}_{43}$ & $\mathrm{SMR}_{53}$ \\
\hline & - & $\mathrm{SMR}_{24}$ & - & $\mathrm{SMR}_{44}$ & - \\
\hline
\end{tabular}

TABLE 3: The parameters of candidate resources.

\begin{tabular}{|c|c|c|c|c|c|c|c|c|c|}
\hline & $C_{1}$ & $C_{2}$ & $T_{1}$ & $T_{2}$ & $Q$ & $\mathrm{RE}$ & $\mathrm{CR}$ & $\mathrm{RM}$ & SE \\
\hline $\mathrm{SMR}_{11}$ & 200 & 190 & 12 & 12 & 0.97 & 0.92 & 0.85 & 9 & 3 \\
\hline $\mathrm{SMR}_{12}$ & 210 & 170 & 13 & 15 & 0.95 & 0.89 & 0.85 & 8 & 5 \\
\hline $\mathrm{SMR}_{13}$ & 240 & 160 & 15 & 10 & 0.99 & 0.88 & 0.93 & 9 & 5 \\
\hline $\mathrm{SMR}_{21}$ & 30 & 30 & 20 & 19 & 0.90 & 0.86 & 0.89 & 6 & 5 \\
\hline $\mathrm{SMR}_{22}$ & 20 & 20 & 10 & 5 & 0.98 & 0.91 & 0.97 & 8 & 2 \\
\hline $\mathrm{SMR}_{23}$ & 10 & 12 & 25 & 13 & 0.92 & 0.91 & 0.87 & 6 & 2 \\
\hline $\mathrm{SMR}_{24}$ & 40 & 19 & 5 & 16 & 0.94 & 0.96 & 0.93 & 8 & 2 \\
\hline $\mathrm{SMR}_{31}$ & 220 & 180 & 13 & 20 & 0.99 & 0.87 & 0.95 & 8 & 2 \\
\hline $\mathrm{SMR}_{32}$ & 200 & 170 & 2 & 3 & 0.95 & 0.92 & 0.98 & 9 & 3 \\
\hline $\mathrm{SMR}_{33}$ & 190 & 190 & 19 & 15 & 0.92 & 0.95 & 0.89 & 8 & 3 \\
\hline $\mathrm{SMR}_{41}$ & 50 & 50 & 9 & 13 & 0.94 & 0.85 & 0.89 & 8 & 4 \\
\hline $\mathrm{SMR}_{42}$ & 70 & 40 & 3 & 5 & 0.91 & 0.89 & 0.87 & 7 & 3 \\
\hline $\mathrm{SMR}_{43}$ & 40 & 65 & 13 & 19 & 0.90 & 0.93 & 0.96 & 6 & 2 \\
\hline $\mathrm{SMR}_{44}$ & 40 & 50 & 20 & 10 & 0.98 & 0.95 & 0.94 & 8 & 4 \\
\hline $\mathrm{SMR}_{51}$ & 40 & 70 & 4 & 15 & 0.90 & 0.96 & 0.96 & 8 & 3 \\
\hline $\mathrm{SMR}_{52}$ & 50 & 50 & 9 & 19 & 0.96 & 0.96 & 0.93 & 9 & 3 \\
\hline $\mathrm{SMR}_{53}$ & 20 & 40 & 5 & 8 & 0.95 & 0.94 & 0.93 & 8 & 3 \\
\hline
\end{tabular}

TABLE 4: The linking cost of candidate resources.

\begin{tabular}{lcccc}
\hline & $\mathrm{SMR}_{(\mathrm{i}+1) 1}$ & $\mathrm{SMR}_{(\mathrm{i}+1) 2}$ & $\mathrm{SMR}_{(\mathrm{i}+1) 3}$ & $\mathrm{SMR}_{(\mathrm{i}+1) 4}$ \\
\hline $\mathrm{SMR}_{11}$ & 60 & 40 & 60 & 60 \\
$\mathrm{SMR}_{12}$ & 40 & 50 & 40 & 90 \\
$\mathrm{SMR}_{13}$ & 30 & 90 & 30 & 80 \\
$\mathrm{SMR}_{21}$ & 60 & 90 & 60 & - \\
$\mathrm{SMR}_{22}$ & 50 & 50 & 80 & - \\
$\mathrm{SMR}_{23}$ & 90 & 60 & 90 & - \\
$\mathrm{SMR}_{24}$ & 80 & 70 & 20 & 50 \\
$\mathrm{SMR}_{31}$ & 80 & 50 & 50 & 70 \\
$\mathrm{SMR}_{32}$ & 50 & 90 & 80 & - \\
$\mathrm{SMR}_{33}$ & 90 & 50 & 50 & - \\
$\mathrm{SMR}_{41}$ & 90 & 40 & 80 & - \\
$\mathrm{SMR}_{42}$ & 40 & 90 & 50 & - \\
$\mathrm{SMR}_{43}$ & 50 & 50 & 80 & 40 \\
$\mathrm{SMR}_{44}$ & 100 & & & - \\
\hline
\end{tabular}

5.3. Result Verification. In this paper, the enumeration method is used to verify the results. Enumeration method is employed to list all 432 allocations. $C_{\max } / C, T_{\max } / T$ and $Q / Q_{\min }$ are calculated by using formula (8)-(10). The nondominant ranking divides the allocations into 17 levels, and the optimal allocations of the first and second levels are shown in Table 7.

The algorithm is run 10 times when the population size is set to $30,50,80,100,130,150,170,180,190$, and 200 , respectively. The algorithms stop after maximal number of generations, which is set to 200 . The population obtained in each run is recorded, and the data is analyzed and processed to delete duplicate individuals in the population. They are compared with the data in Table 7 . Figure 6 is composed of a scatter chart and a line chart. The scatter chart shows the probability that the data obtained in 10 runs under different population sizes are duplicated with the data in Table 7 , and the line chart shows the evolution of the mean probability of the 10 runs versus the population sizes. In addition, Figure 7 shows the evolution of the average of objective function values in 10 runs versus the population sizes. It is clear from Figures 6 and 7 that the result has good convergence after the population size reaches 180 . 
TABLE 5: The linking time of candidate resources.

\begin{tabular}{|c|c|c|c|c|}
\hline & $\operatorname{SMR}_{(\mathrm{i}+1) 1}$ & $\operatorname{SMR}_{(\mathrm{i}+1) 2}$ & $\operatorname{SMR}_{(\mathrm{i}+1) 3}$ & $\operatorname{SMR}_{(\mathrm{i}+1) 4}$ \\
\hline $\mathrm{SMR}_{11}$ & 9 & 4 & 5 & 4 \\
\hline $\mathrm{SMR}_{12}$ & 5 & 11 & 9 & 8 \\
\hline $\mathrm{SMR}_{13}$ & 4 & 3 & 4 & 5 \\
\hline $\mathrm{SMR}_{21}$ & 9 & 1 & 3 & - \\
\hline $\mathrm{SMR}_{22}$ & 5 & 10 & 5 & - \\
\hline $\mathrm{SMR}_{23}$ & 4 & 4 & 1 & - \\
\hline $\mathrm{SMR}_{24}$ & 10 & 5 & 2 & - \\
\hline $\mathrm{SMR}_{31}$ & 8 & 10 & 1 & 4 \\
\hline $\mathrm{SMR}_{32}$ & 5 & 3 & 3 & 5 \\
\hline $\mathrm{SMR}_{33}$ & 2 & 8 & 10 & 9 \\
\hline $\mathrm{SMR}_{41}$ & 5 & 2 & 2 & - \\
\hline $\mathrm{SMR}_{42}$ & 3 & 9 & 11 & - \\
\hline $\mathrm{SMR}_{43}$ & 10 & 1 & 3 & - \\
\hline $\mathrm{SMR}_{44}$ & 9 & 12 & 9 & - \\
\hline
\end{tabular}

TABLE 6: The allocation of shared manufacturing resources and tasks.

\begin{tabular}{|c|c|c|c|c|c|c|c|c|}
\hline $\mathrm{SMT}_{1}$ & $\mathrm{SMT}_{2}$ & $\mathrm{SMT}_{3}$ & $\mathrm{SMT}_{4}$ & $\mathrm{SMT}_{5}$ & $C_{\max } / \mathrm{C}$ & $T_{\max } / T$ & $Q / Q_{\min }$ & $f$ \\
\hline $\mathrm{SMR}_{13}$ & $\mathrm{SMR}_{22}$ & $\mathrm{SMR}_{32}$ & $\mathrm{SMR}_{44}$ & $\mathrm{SMR}_{52}$ & 1.41 & 1.50 & 1.62 & 0.71 \\
\hline $\mathrm{SMR}_{13}$ & $\mathrm{SMR}_{22}$ & $\mathrm{SMR}_{32}$ & $\mathrm{SMR}_{44}$ & $\mathrm{SMR}_{53}$ & 1.50 & 1.74 & 1.62 & 0.68 \\
\hline $\mathrm{SMR}_{11}$ & $\mathrm{SMR}_{24}$ & $\mathrm{SMR}_{32}$ & $\mathrm{SMR}_{42}$ & $\mathrm{SMR}_{51}$ & 1.39 & 2.17 & 1.56 & 0.67 \\
\hline $\mathrm{SMR}_{12}$ & $\mathrm{SMR}_{22}$ & $\mathrm{SMR}_{32}$ & $\mathrm{SMR}_{44}$ & $\mathrm{SMR}_{53}$ & 1.58 & 1.59 & 1.60 & 0.65 \\
\hline $\mathrm{SMR}_{12}$ & $\mathrm{SMR}_{23}$ & $\mathrm{SMR}_{32}$ & $\mathrm{SMR}_{44}$ & $\mathrm{SMR}_{53}$ & 1.60 & 1.42 & 1.58 & 0.61 \\
\hline $\mathrm{SMR}_{11}$ & $\mathrm{SMR}_{22}$ & $\mathrm{SMR}_{32}$ & $\mathrm{SMR}_{42}$ & $\mathrm{SMR}_{53}$ & 1.50 & 2.15 & 1.59 & 0.59 \\
\hline $\mathrm{SMR}_{13}$ & $\mathrm{SMR}_{22}$ & $\mathrm{SMR}_{32}$ & $\mathrm{SMR}_{42}$ & $\mathrm{SMR}_{53}$ & 1.43 & 2.15 & 1.59 & 0.58 \\
\hline $\mathrm{SMR}_{11}$ & $\mathrm{SMR}_{22}$ & $\mathrm{SMR}_{32}$ & $\mathrm{SMR}_{41}$ & $\mathrm{SMR}_{53}$ & 1.53 & 2.00 & 1.60 & 0.56 \\
\hline $\mathrm{SMR}_{13}$ & $\mathrm{SMR}_{22}$ & $\mathrm{SMR}_{32}$ & $\mathrm{SMR}_{41}$ & $\mathrm{SMR}_{53}$ & 1.45 & 2.00 & 1.60 & 0.55 \\
\hline
\end{tabular}

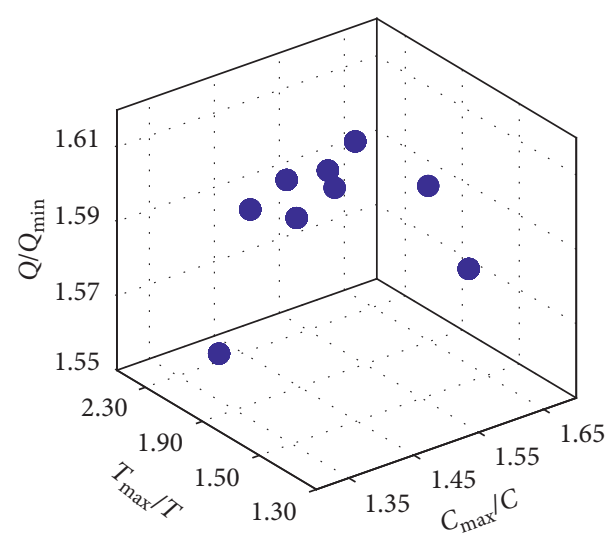

Figure 5: Plot of the final populations with the Maximum fitness value found by Improved NSGA-II.

Similarly, the algorithm is run 10 times when the number of generations is set to $50,100,150,180,190,200,210,220$, and 230, respectively. According to the previous discussion, the population size is set to 180 . The population obtained in each run is recorded, and the data is analyzed and processed to delete duplicate individuals in the population. They are compared with the data in Table 7. Figure 8 is composed of a scatter chart and a line chart. The scatter chart shows the probability that the data obtained in 10 runs under different numbers of generations are duplicated with the data in Table 7 , and the line chart shows the evolution of the mean probability of the 10 runs versus the numbers of generations. Moreover, Figure 9 shows the evolution of the average of objective function values in 10 runs versus the numbers of generations. It is clear that the result has good convergence after the number of generations reaches 200 from Figures 8 and 9. 
TABLE 7: The allocation in the first two levels of enumeration.

\begin{tabular}{|c|c|c|c|c|}
\hline $\mathrm{SMT}_{1}$ & $\mathrm{SMT}_{2}$ & $\mathrm{SMT}_{3}$ & $\mathrm{SMT}_{4}$ & $\mathrm{SMT}_{5}$ \\
\hline $\mathrm{SMR}_{11}$ & $\mathrm{SMR}_{22}$ & $\mathrm{SMR}_{31}$ & $\mathrm{SMR}_{44}$ & $\mathrm{SMR}_{53}$ \\
\hline $\mathrm{SMR}_{11}$ & $\mathrm{SMR}_{22}$ & $\mathrm{SMR}_{32}$ & $\mathrm{SMR}_{41}$ & $\mathrm{SMR}_{53}$ \\
\hline $\mathrm{SMR}_{11}$ & $\mathrm{SMR}_{22}$ & $\mathrm{SMR}_{32}$ & $\mathrm{SMR}_{42}$ & $\mathrm{SMR}_{51}$ \\
\hline $\mathrm{SMR}_{11}$ & $\mathrm{SMR}_{22}$ & $\mathrm{SMR}_{32}$ & $\mathrm{SMR}_{42}$ & $\mathrm{SMR}_{53}$ \\
\hline $\mathrm{SMR}_{11}$ & $\mathrm{SMR}_{22}$ & $\mathrm{SMR}_{32}$ & $\mathrm{SMR}_{44}$ & $\mathrm{SMR}_{53}$ \\
\hline $\mathrm{SMR}_{12}$ & $\mathrm{SMR}_{23}$ & $\mathrm{SMR}_{32}$ & $\mathrm{SMR}_{44}$ & $\mathrm{SMR}_{53}$ \\
\hline $\mathrm{SMR}_{13}$ & $\mathrm{SMR}_{22}$ & $\mathrm{SMR}_{31}$ & $\mathrm{SMR}_{44}$ & $\mathrm{SMR}_{52}$ \\
\hline $\mathrm{SMR}_{13}$ & $\mathrm{SMR}_{22}$ & $\mathrm{SMR}_{31}$ & $\mathrm{SMR}_{44}$ & $\mathrm{SMR}_{53}$ \\
\hline $\mathrm{SMR}_{13}$ & $\mathrm{SMR}_{22}$ & $\mathrm{SMR}_{32}$ & $\mathrm{SMR}_{41}$ & $\mathrm{SMR}_{53}$ \\
\hline $\mathrm{SMR}_{13}$ & $\mathrm{SMR}_{22}$ & $\mathrm{SMR}_{32}$ & $\mathrm{SMR}_{42}$ & $\mathrm{SMR}_{51}$ \\
\hline $\mathrm{SMR}_{13}$ & $\mathrm{SMR}_{22}$ & $\mathrm{SMR}_{32}$ & $\mathrm{SMR}_{42}$ & $\mathrm{SMR}_{53}$ \\
\hline $\mathrm{SMR}_{13}$ & $\mathrm{SMR}_{22}$ & $\mathrm{SMR}_{32}$ & $\mathrm{SMR}_{44}$ & $\mathrm{SMR}_{52}$ \\
\hline $\mathrm{SMR}_{13}$ & $\mathrm{SMR}_{22}$ & $\mathrm{SMR}_{32}$ & $\mathrm{SMR}_{44}$ & $\mathrm{SMR}_{53}$ \\
\hline $\mathrm{SMR}_{11}$ & $\mathrm{SMR}_{22}$ & $\mathrm{SMR}_{32}$ & $\mathrm{SMR}_{44}$ & $\mathrm{SMR}_{52}$ \\
\hline $\mathrm{SMR}_{13}$ & $\mathrm{SMR}_{22}$ & $\mathrm{SMR}_{31}$ & $\mathrm{SMR}_{41}$ & $\mathrm{SMR}_{53}$ \\
\hline $\mathrm{SMR}_{11}$ & $\mathrm{SMR}_{22}$ & $\mathrm{SMR}_{31}$ & $\mathrm{SMR}_{44}$ & $\mathrm{SMR}_{52}$ \\
\hline $\mathrm{SMR}_{11}$ & $\mathrm{SMR}_{22}$ & $\mathrm{SMR}_{31}$ & $\mathrm{SMR}_{41}$ & $\mathrm{SMR}_{53}$ \\
\hline $\mathrm{SMR}_{11}$ & $\mathrm{SMR}_{22}$ & $\mathrm{SMR}_{32}$ & $\mathrm{SMR}_{41}$ & $\mathrm{SMR}_{52}$ \\
\hline $\mathrm{SMR}_{11}$ & $\mathrm{SMR}_{22}$ & $\mathrm{SMR}_{32}$ & $\mathrm{SMR}_{43}$ & $\mathrm{SMR}_{53}$ \\
\hline $\mathrm{SMR}_{11}$ & $\mathrm{SMR}_{24}$ & $\mathrm{SMR}_{32}$ & $\mathrm{SMR}_{41}$ & $\mathrm{SMR}_{53}$ \\
\hline $\mathrm{SMR}_{11}$ & $\mathrm{SMR}_{24}$ & $\mathrm{SMR}_{32}$ & $\mathrm{SMR}_{42}$ & $\mathrm{SMR}_{51}$ \\
\hline $\mathrm{SMR}_{11}$ & $\mathrm{SMR}_{24}$ & $\mathrm{SMR}_{32}$ & $\mathrm{SMR}_{42}$ & $\mathrm{SMR}_{53}$ \\
\hline $\mathrm{SMR}_{12}$ & $\mathrm{SMR}_{22}$ & $\mathrm{SMR}_{31}$ & $\mathrm{SMR}_{44}$ & $\mathrm{SMR}_{53}$ \\
\hline $\mathrm{SMR}_{12}$ & $\mathrm{SMR}_{22}$ & $\mathrm{SMR}_{32}$ & $\mathrm{SMR}_{41}$ & $\mathrm{SMR}_{53}$ \\
\hline $\mathrm{SMR}_{12}$ & $\mathrm{SMR}_{22}$ & $\mathrm{SMR}_{32}$ & $\mathrm{SMR}_{42}$ & $\mathrm{SMR}_{53}$ \\
\hline $\mathrm{SMR}_{12}$ & $\mathrm{SMR}_{22}$ & $\mathrm{SMR}_{32}$ & $\mathrm{SMR}_{44}$ & $\mathrm{SMR}_{53}$ \\
\hline $\mathrm{SMR}_{13}$ & $\mathrm{SMR}_{22}$ & $\mathrm{SMR}_{31}$ & $\mathrm{SMR}_{41}$ & $\mathrm{SMR}_{52}$ \\
\hline $\mathrm{SMR}_{13}$ & $\mathrm{SMR}_{22}$ & $\mathrm{SMR}_{32}$ & $\mathrm{SMR}_{41}$ & $\mathrm{SMR}_{52}$ \\
\hline $\mathrm{SMR}_{13}$ & $\mathrm{SMR}_{22}$ & $\mathrm{SMR}_{32}$ & $\mathrm{SMR}_{42}$ & $\mathrm{SMR}_{52}$ \\
\hline $\mathrm{SMR}_{13}$ & $\mathrm{SMR}_{22}$ & $\mathrm{SMR}_{32}$ & $\mathrm{SMR}_{43}$ & $\mathrm{SMR}_{53}$ \\
\hline $\mathrm{SMR}_{13}$ & $\mathrm{SMR}_{24}$ & $\mathrm{SMR}_{32}$ & $\mathrm{SMR}_{41}$ & $\mathrm{SMR}_{53}$ \\
\hline $\mathrm{SMR}_{13}$ & $\mathrm{SMR}_{24}$ & $\mathrm{SMR}_{32}$ & $\mathrm{SMR}_{42}$ & $\mathrm{SMR}_{53}$ \\
\hline $\mathrm{SMR}_{13}$ & $\mathrm{SMR}_{24}$ & $\mathrm{SMR}_{32}$ & $\mathrm{SMR}_{44}$ & $\mathrm{SMR}_{53}$ \\
\hline
\end{tabular}

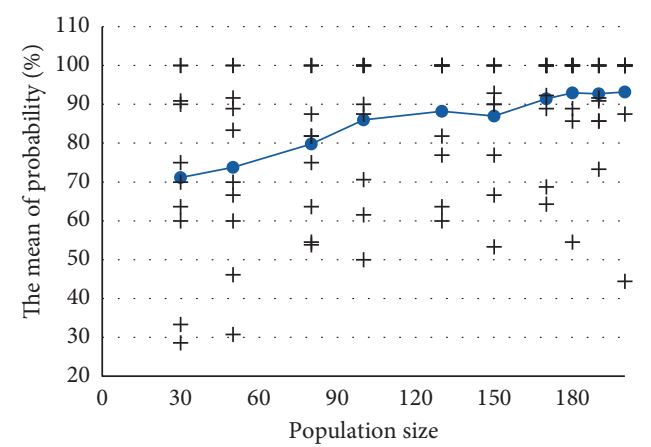

Figure 6: Evolution of the mean of probability of individuals in the first two levels of enumeration in the population in 10 runs versus the population sizes when the number of generations is 200 . 


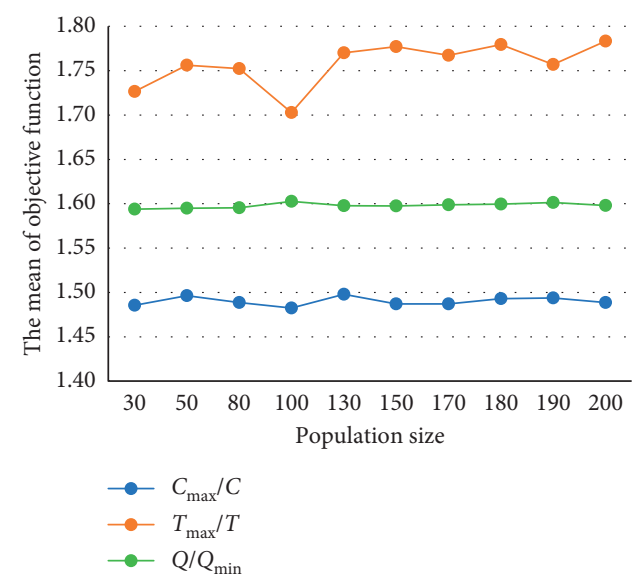

FIGURE 7: Evolution of the mean of objective function values in 10 runs versus the population sizes when the number of generations is 200.

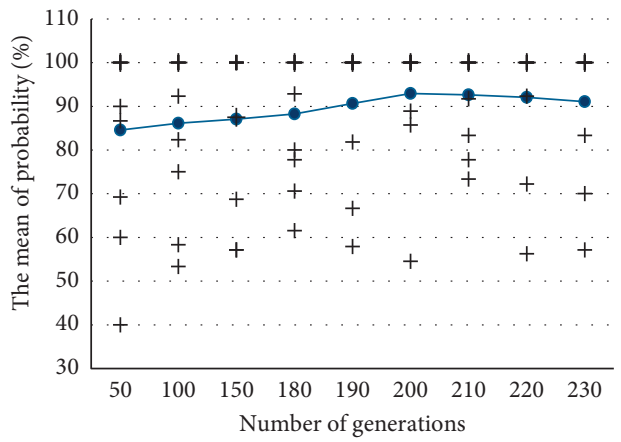

FIGURE 8: Evolution of the mean of probability of individuals in the first two levels of enumeration in the population in 10 runs versus the numbers of generations when the population size is 180 .

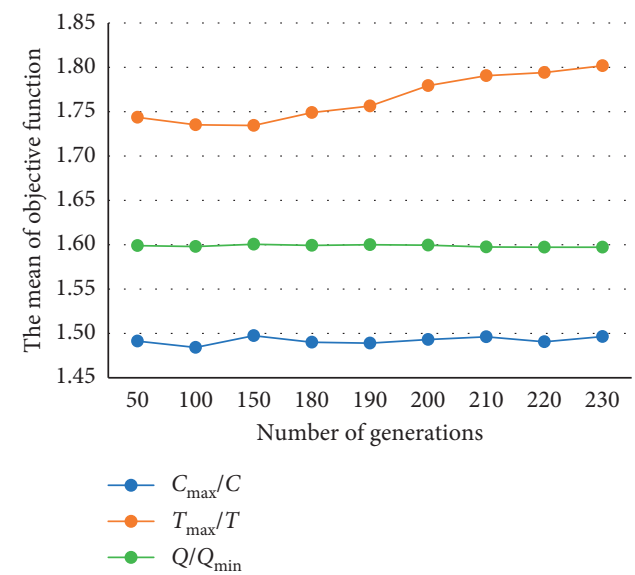

Figure 9: Evolution of the mean of objective function values in 10 runs versus the numbers of generations when the population size is 180.

\section{Conclusions and Managerial Insights}

In this paper, the optimal allocation of manufacturing resources in the shared manufacturing environment is studied, and the process of the shared manufacturing resource allocation is analyzed on the basis of the introduction of the shared process. Considering the interests of consumers and the shared manufacturing platform, the shared manufacturing platform and consumers are the upper and lower decision-making bodies. Taking the reliability, credibility, risk management, and service evaluation as the upper-level optimization indicators representing the platform's rights and interests, and taking the time, cost, and quality qualification rate as the lower-level optimization indicators representing the consumers' rights and interests, a bilevel programming model for optimal allocation of shared 
manufacturing resources is established. Using AHP and Improved G1 combination method to give weight to the upper indexes, the whole bilevel programming model is solved by Improved NSGA-II. The demonstration results of the numerical example show that the shared manufacturing resource allocation can be effectively solved by the resource allocation method in this paper.

Through the analysis of the model results, we got some important managerial insights as follows. On the one hand, we should improve the construction of the monitoring chain and strengthen the supervision and control of the configuration chain, the service chain, and the payment chain. Firstly, the agents of resources and tasks should be checked to ensure the fairness and rationality. Secondly, it is wise to pay attention to whether the providers are in compliance. Thirdly, the rate of payment should be focused to protect the interests of providers. Therefore, the construction of the monitoring chain is necessary. On the other hand, flexible indicators and QoS indicators as important factors in decision-making should be further strengthened. That is, the role of reliability indicator, credibility indicator, credibility indicator, risk management indicator, and service evaluation indicator should be emphasized to ensure the completion of tasks. At the same time, the control of cost, time, and quality qualification should be another part that needs attention.

The future work of this research is as follows. Firstly, the shared manufacturing resource allocation is going to be discussed by considering the situation of resources entering and exiting. Secondly, it is wise to analyze the shared manufacturing resource allocation considering the change of task's requirements. Thirdly, the other intelligent optimization algorithm will be developed to address the shared manufacturing resource allocation. Fourthly, the shared manufacturing resource allocation considering lower-level indicators with unequal weights will be studied.

\section{Data Availability}

The data that support the findings of this study are available upon request from the corresponding author.

\section{Conflicts of Interest}

The authors declare that they have no conflicts of interest.

\section{Acknowledgments}

This work was supported by the Philosophy and Social Science Planning Project of Shenyang under Grant SY202106L. The authors wish to acknowledge the contribution of Liaoning Key Lab of Equipment Manufacturing Engineering Management, Liaoning Research Base of Equipment Manufacturing Development, Liaoning Key Research Base of Humanities and Social Sciences: Research Center of Micro-Management Theory of SUT.

\section{References}

[1] B. Ellen, "A vision for shared manufacturing," Mechanical Engineering, vol. 112, pp. 52-55, 1990.
[2] T. M. A. Ari Samadhi and K. Hoang, "Shared computerintegrated manufacturing for various types of production environment," International Journal of Operations and Production Management, vol. 15, no. 5, pp. 95-108, 1995.

[3] M. Sheikhzadeh, S. Benjaafar, and D. Gupta, "Machine sharing in manufacturing systems: total flexibility versus chaining," International Journal of Flexible Manufacturing Systems, vol. 10, no. 4, pp. 351-378, 1998.

[4] C. Yu, X. Xu, S. Yu, Z. Sang, C. Yang, and X. Jiang, "Shared manufacturing in the sharing economy: concept, definition and service operations," Computers and Industrial Engineering, vol. 146, Article ID 106602, 2020.

[5] P. Jiang and P. Li, "Shared factory: a new production node for social manufacturing in the context of sharing economy," Proceedings of the Institution of Mechanical Engineers-Part B: Journal of Engineering Manufacture, vol. 234, no. 6, pp. 285-294, 2019.

[6] K. Li, W. Xiao, and X. Zhu, "Pricing strategies for sharing manufacturing model based on the cloud platform," Control and Decision, 2021, in press.

[7] Y. Xu, R. Zhi, F. Zheng, and M. Liu, "Online Strategy and competitive analysis of production order scheduling problem with rental cost of shared machines," Chinese Journal of Management Science, 2021, in press.

[8] C. Yu, X. Jiang, S. Yu, and C. Yang, "Blockchain-based shared manufacturing in support of cyber physical systems: concept, framework, and operation," Robotics and Computer-Integrated Manufacturing, vol. 64, Article ID 101931, 2020.

[9] N. Rozman, J. Diaci, and M. Corn, "Scalable framework for blockchain-based shared manufacturing," Robotics and Computer-Integrated Manufacturing, vol. 71, Article ID 102139, 2021.

[10] J. He, J. Zhang, and X. Gu, "Research on sharing manufacturing in Chinese manufacturing industry," International Journal of Advanced Manufacturing Technology, vol. 104, no. 1-4, pp. 463-476, 2019.

[11] P. Li and P. Jiang, "Enhanced agents in shared factory: enabling high-efficiency self-organization and sustainability of the shared manufacturing resources," Journal of Cleaner Production, vol. 292, Article ID 126020, 2021.

[12] G. Wang, G. Zhang, X. Guo, and Y. Zhang, "Digital twindriven service model and optimal allocation of manufacturing resources in shared manufacturing," Journal of Manufacturing Systems, vol. 59, pp. 165-179, 2021.

[13] N. F. Ayala, C. A. Paslauski, A. Ghezzi, and A. G. Frank, "Knowledge sharing dynamics in service suppliers' involvement for servitization of manufacturing companies," International Journal of Production Economics, vol. 193, pp. 538-553, 2017.

[14] D. Zhao and Z. Wang, "A multi-structure service composition of manufacturing resource allocation under the mode of cloud manufacturing," Industrial Engineering Journal, vol. 22, no. 1, pp. 1-10, 2019.

[15] Y. Chen, K. Duan, J. Liu, and L. Wang, "Manufacturing resource optimization allocation model based on bi-level programming in cloud manufacturing," Application Research of Computers, vol. 36, no. 12, pp. 3713-3717, 2019.

[16] J. Zhao, X. Wang, W. Guan, and L. Yin, "Selection of cloud manufacturing services considering psychology based on bilevel programming," Application Research of Computers, vol. 35, no. 2, pp. 594-599, 2018.

[17] P. Wang, H. Xiao, and Y. Pan, "Cloud manufacturing resource service composition based on bi-level programming," Computer Integrated Manufacturing Systems, 2021, in press. 
[18] K. Su, W. Xu, and J. Li, "Manufacturing resource allocation method based on bi-level programming in cloud manufacturing," Computer Integrated Manufacturing Systems, vol. 21, no. 7, pp. 1941-1952, 2015.

[19] Y. Wu, G. Jia, S. Luan, L. Wang, and Y. Gao, "Resource allocation model based on cloud manufacturing," Systems Engineering, vol. 36, no. 3, pp. 122-128, 2018.

[20] H. Wang and G. Du, "Hierarchical joint optimization for design and implementation of shared -service software products," Industrial Engineering and Management, vol. 24, no. 5, pp. 138-145, 2019.

[21] J. Luo and Q. Wu, "Product services system configuration under perspective of supply-demand interaction," Computer Integrated Manufacturing Systems, vol. 26, no. 5, pp. 13041313, 2020.

[22] W. Wang, H. Zhang, L. Ding, W. Zhang, G. Gao, and H. Zhang, "Transportation network optimization of the hazardous materials considering vehicle speed limit interval," Operations Research and Management Science, vol. 20, no. 4, pp. 128-134, 2021.

[23] L. Huang and Y. Ji, "A bi-level optimization model for logistics distribution center location problem under customer delay cost," Industrial Engineering Journal, vol. 24, no. 2, pp. 141-147, 2021.

[24] Y. Dong, Q. Zheng, and L. Li, "Bi-level programming model for multi-source emergency materials procurement considering supply risks," Chinese Journal of Management Science, vol. 29, no. 11, pp. 170-178, 2021.

[25] S. Wang, W. Song, L. Kang, Q. Li, L. Guo, and G. Chen, "Manufacturing resource allocation based on cloud manufacturing," Computer Integrated Manufacturing Systems, vol. 18, no. 7, pp. 1396-1405, 2012.

[26] C. $\mathrm{Xu}$ and F. Li, "A research on optimal allocation of manufacturing resources for cloud manufacturing services," Industrial Engineering Journal, vol. 22, no. 3, pp. 44-51, 2019.

[27] X. Gao, Y. Jing, and J. Du, "Optimal allocation method of manufacturing resource based on improved genetic algorithm," Manufacturing Automation, vol. 39, no. 11, pp. 99104, 2017.

[28] J. Yang, M. Luo, and S. Yuan, "An extended global PCP algorithm for linear bi-level programming," Operations Research and Management Science, vol. 30, no. 1, pp. 23-28, 2021. 